\title{
Evaluación de la salud forestal de una plantación de Eucalyptus globulus Labill con fines de manejo en la Estación Experimental Tunshi, Riobamba, Ecuador
}

\author{
Miguel Ángel Guallpa Calva, Mgs \\ Víctor Manuel Espinoza, Mgs \\ Edmundo Danilo Guilcapi Pacheco, Mgs \\ Hernán Eriberto Chamorro Sevilla, Mgs
}

Facultad de Recursos Naturales, Escuela Superior Politécnica de

Chimborazo, Riobamba, Ecuador

Doi:10.19044/esj.2019.v15n21p104 URL:http://dx.doi.org/10.19044/esj.2019.v15n21p104

\section{Resume}

Frente a la necesidad de indagar sobre la salud forestal de una plantación de Eucalyptus globulus Labill con el propósito de disponer de información complementaria, que oriente la planificación de actividades silvícolas para su conservación y aprovechamiento, es la razón que motivó evaluar la condición de salud de los árboles en pie por rodal. Mediante el muestreo sistemático no alineado a una intensidad del 5-6\% ha ${ }^{-1}$, en base a la instalación de parcelas circulares, dentro de cada parcela se registró seis rasgos que reflejan el estado de vigorosidad, saludable, enfermedad, debilidad, moribundo y árbol muerto en pie, mismos que indican el nivel de pérdida de hojas en los árboles. La aplicación de tablas de contingencia para el análisis de los residuos corregidos, siendo significativo al 5\%, si el valor absoluto es $>1,96$. Los resultados obtenidos mostraron cinco asociaciones significativas a excepción del rasgo árbol muerto en pie. Finalmente, con la formación de dos conglomerados jerárquicos que agrupa el nivel de salud, la decisión para la gestión del bosque plantado de eucalipto comprende el aprovechamiento de los rodales 2, 5, 6 y 7, más el manejo posterior de los rebrotes resultantes y de carácter conservacionista a los rodales 1, 3 y 4. La decisión se enmarca bajo criterios de manejo sostenible de los recursos forestales en base a los indicadores de salud que presentó el bosque plantado.

Palabras clave: Salud forestal, Rodal, Bosque plantado, Eucalyptus globulus 


\title{
Forest Health Evaluation of an Eucalyptus Globulus Labill Plantation for Handling Purposes at Tunshi Experimental Station, Riobamba, Ecuador
}

\author{
Miguel Ángel Guallpa Calva, Mgs \\ Víctor Manuel Espinoza, Mgs \\ Edmundo Danilo Guilcapi Pacheco, Mgs \\ Hernán Eriberto Chamorro Sevilla, Mgs
}

Facultad de Recursos Naturales, Escuela Superior Politécnica de

Chimborazo, Riobamba, Ecuador

\begin{abstract}
Faced with the need to inquire on the forest health of an Eucalyptus globulus Labill plantation with the purpose of providing complementary information, which guides the planning of silvicultural activities for its conservation and use, is the reason that motivated to evaluate the health of the trees standing by stand. Through the systematic sampling not aligned at an intensity of 5-6\% ha $\mathrm{h}^{-1}$, based on the installation of circular plots, within each plot, six features were recorded that reflect the state of vigor, health, disease, weakness, dying and standing dead tree, which indicate the level of loss leaves in trees. The application of contingency tables for the analysis of corrected waste, being $5 \%$, so the absolute value is $>1,96$. The results obtained showed five significant associations except for the dead tree feature. Finally, with the formation of two hierarchical clusters by grouping the state of forest health of the eucalyptus plantation, reinforces the decision form for the management on the planted forest considering the execution use of 2, 5, 6 and 7 stands, also the subsequent management of the resulting and conservationist sprouts to stands 1,3 and 4 . The decision was taken under the criteria of sustainable management of forest resources, which is based on the productive and health indicators presented by the planted forest.
\end{abstract}

Keywords: Forest health, Stand, Planted forest, Eucalyptus globulus

\section{Introducción}

Los bosques plantados se encuentran sujetos a varias alteraciones que pueden ser naturales o antrópicas, en el primer caso sequias, deslizamientos de tierras, insectos dañinos, brotes de enfermedades, fenómenos climáticos; y 
en el segundo caso incendios, introducción de especies invasoras y el mal manejo; están a su vez, fuertemente influidas por las características del sitio que inciden en la composición, estructura y funciones de los bosques (Dale et al., 2001).

El eucalipto es una especie de alta capacidad productiva que prospera en terrenos forestales degradados o inutilizados, desarrollando en condiciones de suelo y clima diversas (ENCE, 2009). En Ecuador, el E. globulus es priorizado en la región sierra para la forestación con fines comerciales, la protección de suelos degradados, debido a su adaptabilidad, rápido crecimiento y rentabilidad (MAGAP, 2015). Siendo evidente, cuando se aplica estrategias silvícolas orientadas al manejo sostenible de la especie forestal en estudio, con el fin de obtener una óptima y continua productividad del sitio (Hernández, 2014).

$\mathrm{Su}$ rentabilidad, se refleja por los niveles de venta de varios productos de madera aserrada, y residuos forestales de eucalipto que se generan en los aserraderos, los cuales son mayormente utilizados en los cantones Riobamba, Ambato y Latacunga con el 34, 34.48 y $41.03 \%$ respectivamente de acuerdo a lo reportado por Guallpa, Rosero, Montenegro, \& Quinchuela. (2018). Por tanto, es imperante disponer de información técnica de los parámetros de rendimiento, el estado de salud de los árboles, así como las posibles causas que influyen en la calidad de la madera en pie (Rojas \& Murillo, 2000), y en caso de no tener datos precisos de su condición de salud actual, es necesario realizar el monitoreo para detectar cambios eventuales o permanentes en el recurso (Saavedra et al., 2016). Debido a la interacción entre las condiciones del sitio, la especie utilizada, edad, del uso de material genético de calidad, la compactación del suelo y del manejo silvicultural aplicado a la plantación, el cual incide en la calidad de los árboles que conforman los rodales de una masa forestal y la calidad de madera a obtener (Murillo \& Badilla, 2010; Scharenbroch, Lloyd, \& Johnson, 2005). De acuerdo a lo expuesto, la valoración de salud de los rodales es requerida para la generación esquemas de manejo silvícola con fines de conservación y aprovechamiento (Jiménez, 2008).

En la provincia de Chimborazo, la mayoría de evaluaciones de plantaciones forestales están orientadas específicamente al cálculo del volumen de madera en pie, y en ciertos casos la información de calidad es manejada de manera particular por ciertas empresas o propietarios de masas forestales. La plantación en estudio es parte de la Estación Experimental Tunshi de la Escuela Superior Politécnica de Chimborazo, entre sus principales funciones: por una parte, contribuye a la generación de microclima y por otra contribuye en la generación de espacios para la realización de prácticas e investigaciones académicas (Guallpa, Lara, Espinoza, Guilcapi, \& Fosado, 2019). 
Generalmente, los primeros síntomas de deterioro del árbol se expresan en la apariencia de sus hojas y copas (Schomaker et al., 2007); los árboles con altas proporciones a nivel de su crecimiento en altura y copa viva, diámetros grandes, altas densidades de copa, tienen altas tasas de crecimiento y mayores probabilidades de sobrevivencia (Awal et al., 2010). Las copas densamente foliadas están asociadas con árboles vigorosos, mientras que las pequeñas y de follaje disperso podrían encontrarse en un estado de debilitamiento (Shomaker et al., 2007); por lo tanto, a través de su evaluación, fue importante caracterizar la salud de los árboles en 7 rodales de la plantación en estudio. A fin de complementar y disponer de información sólida que servirá de base para una mejor planificación, la toma de decisiones adecuadas para obtener mejores resultados como parte del manejo de la plantación.

Partiendo del hecho que la productividad y salud de los árboles de eucalipto a nivel de sitio y micro sitio en el sector de Tunshi, en condiciones adversas, y la ausencia de actividades silviculturales ejercen influencia sobre la salud de los árboles en pie, se decidió valorar la masa forestal, para disponer de su caracterización cualitativa como soporte complementario para la toma de decisiones de manejo técnico (Guallpa, Rosero, Samaniego, \& Cevallos, 2016).

En base al inventario por muestreo de la plantación, se determinó la información considerando una variable clasificatoria del estado de salud de los árboles, misma que agrupa a seis variables seleccionadas (Nájera \& Hernández, 2008). El objetivo del presente estudio fue valorar seis rasgos de salud de los árboles en pie por rodal. A fin de disponer, de indicadores técnicos que orienten el manejo sostenible del bosque plantado en estudio.

\section{Metodología}

La investigación se llevó a cabo en la plantación de E. globulus Labill de 38 años de edad, ubicada en la Estación Experimental Tunshi de la Escuela Superior Politécnica de Chimborazo, Tunshi Grande, parroquia Licto, al sur oeste del cantón Riobamba, provincia de Chimborazo-Ecuador, a $12 \mathrm{~km}$ de la ciudad capital. Geográficamente la masa forestal se inserta dentro de los puntos de referencia: UTM Zona 17S Datum WGS $84 \mathrm{X}_{1}=763609 ; \mathrm{Y}_{1}=$ 9806880, $\mathrm{X}_{2}=764973 ; \mathrm{Y}_{2}=9806791, \mathrm{X}_{3}=764973 ; \mathrm{Y}_{3}=9805248$ y $\mathrm{X}_{4}=$ 763606; $Y_{4}=9805251$. Se sitúa a una altitud que va desde 2740 - $2929 \mathrm{msnm}$. La precipitación anual es $636,4 \mathrm{~mm}$, siendo la temperatura media $14,5{ }^{\circ} \mathrm{C}$ y una humedad relativa media de $85 \%$. Datos de la Estación Guaslan; serie 1990-2014; MAGAP. El bosque plantado en estudio se categoriza dentro de la formación: estepa espinosa-Montano Bajo (Sierra, Cerón, Palacios, \& Valencia, 1999). 
La Tabla1 muestra las características fisiográficas y del suelo del área de plantación reportadas en el estudio desarrollado por (Guallpa, Rosero, Samaniego, \& Cevallos, 2016).

Tabla 1. Principales características fisiográficas y del suelo en el área de estudio

\begin{tabular}{|c|c|c|l|l|}
\hline Rodal & $\begin{array}{c}\text { Altitud } \\
(\mathbf{m s n m})\end{array}$ & $\begin{array}{c}\text { Pendiente } \\
(\mathbf{\%})\end{array}$ & $\begin{array}{c}\text { Nivel de pedregosidad } \\
\text { interna }(\mathbf{\%})\end{array}$ & $\begin{array}{c}\text { Nivel de drenaje interno } \\
\left(\mathbf{c m} \cdot \mathbf{h}^{-\mathbf{1}}\right)\end{array}$ \\
\hline 1 & 2787 & 20 & No pedregoso & Drenaje moderado \\
\hline 2 & 2861 & 55 & No pedregoso & Drenaje moderadamente lento \\
\hline 3 & 2929 & 55 & No pedregoso & Drenaje moderadamente lento \\
\hline 4 & 2894 & 58 & Moderadamente pedregoso & Drenaje moderadamente lento \\
\hline 5 & 2793 & 57 & No pedregoso & Drenaje moderadamente lento \\
\hline 6 & 2774 & 50 & No pedregoso & Drenaje moderado \\
\hline 7 & 2755 & 18 & Moderadamente pedregoso & Drenaje moderado \\
\hline
\end{tabular}

No pedregosos $=<5 \%$; Moderadamente pedregoso 5-20\%; Drenaje moderadamente lento $=0,51-2,00 \mathrm{~cm} \mathrm{hr}^{-1}$; Drenaje moderado $=2,00-6,30 \mathrm{~cm} \mathrm{hr}^{-1}$

La caracterización de las variables dasométricas de la plantación de $E$. globulus realizada en el año 2014 reportó en estado fustal con un DAP > a 10 $\mathrm{cm}, 441,13$ árboles ha ${ }^{-1}$; un DAP promedio de $23,17 \mathrm{~cm}$, y una altura total de $21,23 \mathrm{~m}$. La media aritmética del área basal de los siete rodales fue de $0,54 \mathrm{~m}^{2}$ árbol ${ }^{-1}$, con 441,13 árboles, se estima $22,21 \mathrm{~m}^{2} \mathrm{ha}^{-1}$ de área basal y una densidad en cobertura del $96,47 \%$ de área basal con un volumen total de

$472,09 \mathrm{~m}^{3} \cdot \mathrm{ha}^{1}$. Adicionalmente en el mismo estudio desarrollado por Guallpa et al. (2016), se determinó la asociación entre rodales, el primer grupo conformado por los rodales 1, 2, 3 y 4 y el segundo por los rodales 5, 6 y 7 .

\section{Área neta de plantación}

El área neta de la masa forestal se determinó mediante tres recorridos tomando puntos geográficos para el cálculo de área y perímetro de cada rodal con un GPS, modelo Garmin 62Csc. Se realizó las post correcciones diferenciales de posicionamiento utilizando sistemas de aumentación SBAS (Satellite-based argumentation system) y finalmente fueron importados a una hoja de cálculo en tablas que contienen los datos de las coordenadas UTM (del inglés, Universal Transverse Mercator) en un formato compatible con el software GIS (del inglés,Geographic Information System). El bosque plantado tiene una superficie total de 38,79 ha, un área sin vegetación de 0,60 ha dando un área neta de 38,19 ha (Figura 1). 


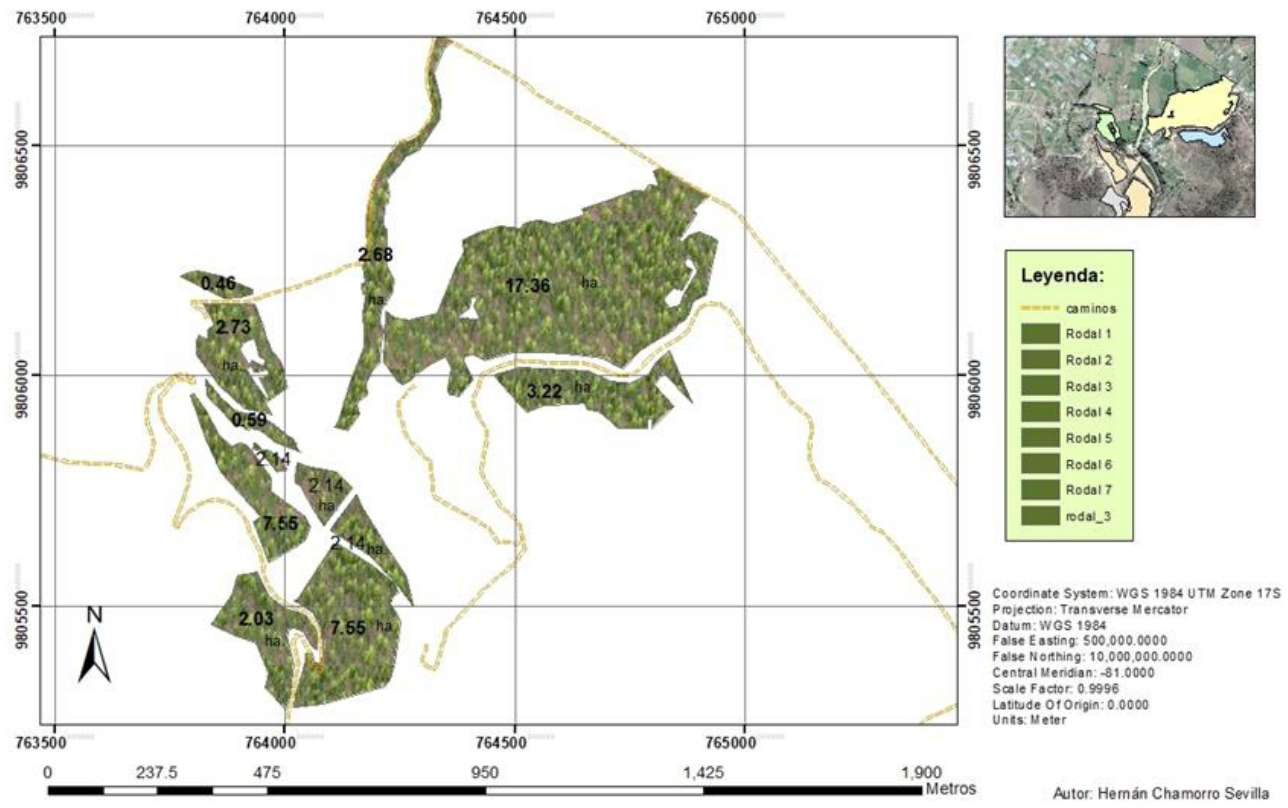

Figura 1. Mapa rodales que integran la plantación de eucalipto en estudio

\section{Muestreo}

El muestreo del estado de salud de la plantación de E. globulus tuvo lugar en el segundo semestre del año 2017. Para evaluar los rodales de la plantación se aplicó un inventario por muestreo sistemático no alineado con una intensidad del 5-6\% ha ${ }^{-1}$, mediante la instalación de parcelas circulares con un radio de 12,62 m, distanciadas entre ellas de 75 y $100 \mathrm{~m}$, de acuerdo a la topografía y exposición de cada rodal (FAO, 2011).

\section{Evaluación de la plantación}

Se fundamentó en la asignación de salud forestal en función de distintas variables cualitativas seleccionadas para determinar las características del estado de los árboles en pie al considerar ciertas variables de la metodología desarrollada por Murillo \& Badilla (2010). Así como en la propuesta de procedimientos para el seguimiento de parcelas permanentes, mediante el registro de criterios considerados en las evaluaciones de campo de los árboles valorados dentro de cada parcela (Salazar, 2008; ETIFN, 2014).

\section{Variable clasificatoria}

El estudio parte de la determinación de los criterios más influyentes en la salud de los árboles en función del rodal en el que se encuentran. La valoración analítica se agrupa en una característica clasificatoria (Tabla 2). Cada árbol es considerado como una "unidad de registro" a la que se le asigna 
una clase de salud. La evaluación se realizó de forma separada para cada una de las características y la subsecuente reagrupación de las valoraciones. Tales criterios, se resume en la Tabla 2, elaborada en base a la clasificación de salud de los árboles, con modificaciones a lo propuesto por la comunidad Europea que se muestra en el trabajo desarrollado sobre declinación forestal (Granados \& López, 2001).

Tabla 2. Variable clasificatoria de calidad de los árboles en pie

\begin{tabular}{|l|l|}
\hline \multicolumn{1}{|c|}{ Atributo } & \multicolumn{1}{c|}{ Descripción } \\
\hline Vigorosidad & Árbol con un nivel de pérdida de hojas de $0-10 \%$ \\
\hline Saludable & Árbol con un nivel de pérdida de hojas de $11-20 \%$ \\
\hline Enfermedad & Árbol con un nivel de pérdida de hojas de 21-50\% \\
\hline Debilidad & Árbol con un nivel de pérdida de hojas de 51-60\% \\
\hline Moribundo & Árbol con un nivel de pérdida de hojas de $60-99 \%$ \\
\hline Muerto en pie & Árbol con un nivel de pérdida de hojas de 100\% \\
\hline
\end{tabular}

Todas estas variables son binarias, donde se asignó el valor 0 que significa ausencia y presencia de la característica evaluada durante la fase de muestreo.

\section{Análisis estadístico}

Se elaboró una base de datos en Excel, utilizada para el análisis individual de cada variable con el objetivo de determinar si existe dependencia o no de cada una de las características con respecto al rodal en el programa estadístico SPSS Statistics 22 (Nel, 2014), mediante tablas de contingencia que incorpora los residuos corregidos y la prueba de Chi-cuadrado de Pearson aplicada a cada análisis para determinar la mayor o menor asociación de cada atributo por rodal, estos métodos permiten, establecer el sentido y quién provoca esa dependencia (De la Fuente-Fernández, 2011).

Los residuos son las diferencias entre la son la frecuencia observada y la frecuencia esperada en cada casilla: $e_{i j}=$ $N_{i j}-E_{i j}$

El análisis de los residuos en las tablas de contingencia, se identifica al estudiar su patrón; pero dado que generalmente no son sencillos de observar se recurre a la comparación de los residuos ajustados a una distribución teórica conocida de acuerdo a lo sugerido por Haberman (1978).

Residuos ajustados

$$
e_{i j}=\frac{N_{i j}-E_{i j}}{\sqrt{E_{i j}}}
$$

$N_{i j}$ : Frecuencia observada.

$E_{i j}$ : Frecuencia teórica.

Residuos tipificados 


$$
\begin{gathered}
d_{i j}=\frac{\left(N_{i j}-E_{i j}\right) / \sqrt{E_{i j}}}{\sqrt{V_{i j}}}=\frac{e_{i j}}{\sqrt{V_{i j}}} \\
V_{i j}=V\left(e_{i j}\right)=\left(1-\frac{n_{i .}}{N}\right)\left(1-\frac{n_{. j}}{N}\right)
\end{gathered}
$$

Cuando los dos factores objeto de estudio son independientes, cada residuo tipificado $\mathrm{d}_{\mathrm{ij}}$ se distribuye asintóticamente como una $\mathrm{N}(0,1)$

1. Para un nivel de significancia del $5 \%$ se comparan los valores absolutos de la tabla de entrada de los residuos corregidos. Es significativo siempre que su valor absoluto supere 1,96.

2. En los valores que cumplen con la condición se analiza el signo, lo que permite conocer el sentido de dicha relación (García, 2016).

Para la interpretación del Test Chi-cuadrado de Pearson, los datos son compatibles con la hipótesis de independencia, si la probabilidad asociada al estadístico $\mathrm{x}^{2}$ es alta cuando el p-valor es $>0,05, \mathrm{y}$ si es<0.05, conduce a rechazar la hipótesis nula, concluyendo que existe dependencia o relación entre las variables analizadas.

También se toma en cuenta, si existen frecuencias esperadas menores que 5, éstas no deben superar el 20 por ciento del total de frecuencias esperadas. Caso contrario se usó el Test de Fisher (López \& Fachelli, 2015).

Finalmente se realizó la reagrupación de las características de salud de los árboles en pie al conformar grupos de rodales con características similares (De la Fuente-Fernández, 2011a). Teniendo en cuenta que no todos los rodales presentan el mismo número de árboles, y que los métodos de clasificación basan su proceder en las distancias entre los valores de cada uno de los criterios, se procedió a trabajar con las frecuencias de presencia de los seis rasgos que conforman el estudio de salud forestal.

\section{Resultados y Discusión}

\section{Estado de salud de los árboles}

\section{Análisis de contingencia de la relación vigorosidad de los árboles en pie} por rodal

El análisis de los residuos corregidos para la variable vigorosidad de los árboles en pie por rodal, refleja que existe dependencia provocada por una tendencia al incremento en los rodales 1 y 5 en sus valores de ausencia $(3,4$ y $2,1)$ y decremento en sus valores de presencia $(-3,4$ y $-2,1)$ en base a la característica evaluada respectivamente. En el caso del rodal 4 ocurre lo contrario, hay un decremento en su valor de ausencia $(-8,8)$ e incremento en su valor de presencia $(8,8$; Tabla 3$)$. 
Tabla 3. Análisis de contingencia de la relación vigorosidad de los árboles en pie por rodal

\begin{tabular}{|c|c|c|c|c|c|}
\hline & \multicolumn{2}{|c|}{ Vigorosidad } & \multirow{2}{*}{ Total } \\
\hline & & & 0 & 1 & \\
\hline & & Recuento & 402 & 0 & 402 \\
\hline & 1 & Frecuencia esperada & 394,8 & 7,2 & 402,0 \\
\hline & & Residuos corregidos & 3,4 & $-3,4$ & \\
\hline \multirow[b]{8}{*}{ Rodal } & \multirow{3}{*}{2} & Recuento & 128 & 0 & 128 \\
\hline & & Frecuencia esperada & 125,7 & 2,3 & 128,0 \\
\hline & & Residuos corregidos & 1,6 & $-1,6$ & \\
\hline & \multirow{3}{*}{3} & Recuento & 32 & 0 & 32 \\
\hline & & Frecuencia esperada & 31,4 & ,6 & 32,0 \\
\hline & & Residuos corregidos & ,8 &,- 8 & \\
\hline & \multirow{3}{*}{4} & Recuento & 210 & 20 & 230 \\
\hline & & Frecuencia esperada & 225,9 & 4,1 & 230,0 \\
\hline & & Residuos corregidos & $-8,8$ & 8,8 & \\
\hline & \multirow{3}{*}{5} & Recuento & 192 & 0 & 192 \\
\hline & & Frecuencia esperada & 188,6 & 3,4 & 192,0 \\
\hline & & Residuos corregidos & 2,1 & $-2,1$ & \\
\hline & \multirow{3}{*}{6} & Recuento & 77 & 0 & 77 \\
\hline & & Frecuencia esperada & 75,6 & 1,4 & 77,0 \\
\hline & & Residuos corregidos & 1,2 & $-1,2$ & \\
\hline & \multirow{3}{*}{7} & Recuento & 53 & 0 & 53 \\
\hline & & Frecuencia esperada & 52,0 & 1,0 & 53,0 \\
\hline & & Residuos corregidos & 1,0 & $-1,0$ & \\
\hline \multirow{2}{*}{\multicolumn{2}{|c|}{ Total }} & Recuento & 1094 & 20 & 1114 \\
\hline & & Frecuencia esperada & 1094,0 & 20,0 & 1114,0 \\
\hline
\end{tabular}

En el caso del análisis de la relación de la variable vigorosidad de los árboles en pie por rodal, el número de casillas con valores esperados inferiores a 5, sobrepasan el $20 \%$ de las frecuencias esperadas (42,9\% de las casillas), por lo que se utilizó el Test de Fisher que sugiere asociación significativa entre la característica vigorosidad de los árboles y los rodales ( $\mathrm{p}$-valor $<\alpha=0.05$; Tabla 4).

Tabla 4. Pruebas de Chi-cuadrado de la tabla de contingencia de la variable vigorosidad de los árboles en pie por rodal

\begin{tabular}{|l|l|l|l|l|l|l|}
\hline & Valor & gl & $\begin{array}{l}\text { Sig. } \\
\text { asintótica } \\
\text { (bilateral) }\end{array}$ & $\begin{array}{l}\text { Sig. exacta } \\
\text { (bilateral) }\end{array}$ & $\begin{array}{l}\text { Sig. exacta } \\
\text { (unilateral) }\end{array}$ & $\begin{array}{l}\text { Probabilidad } \\
\text { en el punto }\end{array}$ \\
\hline Chi-cuadrado de Pearson & $78,275^{\mathrm{a}}$ & 6 & 0,000 & 0,000 & & \\
\hline Razón de verosimilitudes & 64,536 & 6 & 0,000 & 0,000 & & \\
\hline Estadístico exacto de Fisher & 51,356 & & & 0,000 & & \\
\hline Asociación lineal por lineal & $4,193^{\mathrm{b}}$ & 1 & 0,041 & 0,043 & 0,025 & 0,006 \\
\hline N de casos válidos & 1114 & & & & & \\
\hline
\end{tabular}

a. 6 casillas $(42,9 \%)$ tienen una frecuencia esperada inferior a 5. La frecuencia mínima esperada es ,57.

b. El estadístico tipificado es 2,048. 


\section{Análisis de contingencia de la relación árboles saludables en pie por rodal}

El análisis de los residuos corregidos para la variable árboles saludables en pie por rodal, muestra que existe dependencia provocada por una tendencia al incremento en los rodales 1,3 y 4 en sus valores de ausencia $(4,4 ; 3,7$ y 8,1$)$ y decremento en sus valores de presencia $(-4,4 ;-3,7$ y $-8,1)$ en base a la característica evaluada respectivamente. En el caso de los rodales 2 , 5,6 y 7 ocurre lo contrario, hay un decremento en sus valores de ausencia ($3,4 ;-7,8 ;-4,7$ y $-3,8)$ e incremento en sus valores de presencia $(3,4 ; 7,8 ; 4,7 \mathrm{y}$ 3,8; Tabla 5).

Tabla 5. Análisis de contingencia de la relación árboles saludables en pie por rodal

\begin{tabular}{|c|c|c|c|c|c|}
\hline & \multicolumn{2}{|c|}{ Saludables } & \multirow{2}{*}{ Total } \\
\hline & & & 0 & 1 & \\
\hline \multirow{21}{*}{ Rodal } & \multirow{3}{*}{1} & Recuento & 112 & 290 & 402 \\
\hline & & Frecuencia esperada & 83,4 & 318,6 & 402,0 \\
\hline & & Residuos corregidos & 4,4 & $-4,4$ & \\
\hline & \multirow{3}{*}{2} & Recuento & 12 & 116 & 128 \\
\hline & & Frecuencia esperada & 26,5 & 101,5 & 128,0 \\
\hline & & Residuos corregidos & $-3,4$ & 3,4 & \\
\hline & \multirow{3}{*}{3} & Recuento & 15 & 17 & 32 \\
\hline & & Frecuencia esperada & 6,6 & 25,4 & 32,0 \\
\hline & & Residuos corregidos & 3,7 & $-3,7$ & \\
\hline & \multirow{3}{*}{4} & Recuento & 92 & 138 & 230 \\
\hline & & Frecuencia esperada & 47,7 & 182,3 & 230,0 \\
\hline & & Residuos corregidos & 8,1 & $-8,1$ & \\
\hline & \multirow{3}{*}{5} & Recuento & 0 & 192 & 192 \\
\hline & & Frecuencia esperada & 39,8 & 152,2 & 192,0 \\
\hline & & Residuos corregidos & $-7,8$ & 7,8 & \\
\hline & \multirow{3}{*}{6} & Recuento & 0 & 77 & 77 \\
\hline & & Frecuencia esperada & 16,0 & 61,0 & 77,0 \\
\hline & & Residuos corregidos & $-4,7$ & 4,7 & \\
\hline & \multirow{3}{*}{7} & Recuento & 0 & 53 & 53 \\
\hline & & Frecuencia esperada & 11,0 & 42,0 & 53,0 \\
\hline & & Residuos corregidos & $-3,8$ & 3,8 & \\
\hline \multirow{2}{*}{ Total } & & Recuento & 231 & 883 & 1114 \\
\hline & & Frecuencia esperada & 231,0 & 883,0 & 1114,0 \\
\hline
\end{tabular}

Al aplicar el Test Chi-cuadrado de Pearson a los datos obtenidos de la Tabla 5 de contingencia, se rechaza la hipótesis nula y se acepta que hay dependencia entre la variable árboles saludables en pie y los rodales al presentar un $p$-valor $<\alpha=0.05$ como se muestra en la Tabla 6 . 
Tabla 6. Pruebas de Chi-cuadrado de la tabla de contingencia de la variable árboles saludables en pie por rodal

\begin{tabular}{|l|l|l|l|l|l|l|}
\hline & Valor & gl & $\begin{array}{l}\text { Sig. asintótica } \\
\text { (bilateral) }\end{array}$ & $\begin{array}{l}\text { Sig. exacta } \\
\text { (bilateral) }\end{array}$ & $\begin{array}{l}\text { Sig. exacta } \\
\text { (unilateral) }\end{array}$ & $\begin{array}{l}\text { Probabilidad } \\
\text { en el punto }\end{array}$ \\
\hline Chi-cuadrado de Pearson & $171,936^{\mathrm{a}}$ & 6 & 0,000 &.$^{\mathrm{b}}$ & & \\
\hline Razón de verosimilitudes & 228,114 & 6 & 0,000 &.$^{\mathrm{b}}$ & & \\
\hline Estadístico exacto de Fisher &. $\mathrm{b}$ & & & . $^{\mathrm{b}}$ & & \\
\hline Asociación lineal por lineal & $41,190^{\mathrm{c}}$ & 1 & 0,000 & 0,000 & 0,000 & 0,000 \\
\hline N de casos válidos & 1114 & & & & & \\
\hline
\end{tabular}

a. 0 casillas $(0,0 \%)$ tienen una frecuencia esperada inferior a 5. La frecuencia mínima esperada es 6,64.

b. No se puede efectuar el cálculo porque no hay suficiente memoria.

c. El estadístico tipificado es 6,418.

\section{Análisis de contingencia de la relación de la variable enfermedad de los árboles en pie por rodal}

El análisis de los residuos corregidos para la variable enfermedad de los árboles en pie por rodal, refleja dependencia provocada por una tendencia al decremento en el rodal 1 en su valor de ausencia $(-3,4)$ e incremento en su valor de presencia $(3,4)$ del criterio evaluado. En el caso del rodal 5 sucede lo contrario, hay un incremento en su valor de ausencia $(2,0)$ y decremento en su valor de presencia $(-2,0)$ (Tabla 7).

Tabla 7. Análisis de contingencia de la relación de la variable enfermedad de los árboles pie por rodal

\begin{tabular}{|c|c|c|c|c|c|}
\hline & \multicolumn{2}{|c|}{ Enfermedad } & \multirow{2}{*}{ Total } \\
\hline & & & 0 & 1 & \\
\hline \multirow{21}{*}{ Rodal } & \multirow{3}{*}{1} & Recuento & 388 & 14 & 402 \\
\hline & & Frecuencia esperada & 395,1 & 6,9 & 402,0 \\
\hline & & Residuos corregidos & $-3,4$ & 3,4 & \\
\hline & \multirow{3}{*}{2} & Recuento & 127 & 1 & 128 \\
\hline & & Frecuencia esperada & 125,8 & 2,2 & 128,0 \\
\hline & & Residuos corregidos & ,9 &,- 9 & \\
\hline & \multirow{3}{*}{3} & Recuento & 32 & 0 & 32 \\
\hline & & Frecuencia esperada & 31,5 & ,5 & 32,0 \\
\hline & & Residuos corregidos & 8 &,- 8 & \\
\hline & \multirow{3}{*}{4} & Recuento & 226 & 4 & 230 \\
\hline & & Frecuencia esperada & 226,1 & 3,9 & 230,0 \\
\hline & & Residuos corregidos & 0 & ,0 & \\
\hline & \multirow{3}{*}{5} & Recuento & 192 & 0 & 192 \\
\hline & & Frecuencia esperada & 188,7 & 3,3 & 192,0 \\
\hline & & Residuos corregidos & 2,0 & $-2,0$ & \\
\hline & \multirow{3}{*}{6} & Recuento & 77 & 0 & 77 \\
\hline & & Frecuencia esperada & 75,7 & 1,3 & 77,0 \\
\hline & & Residuos corregidos & 1,2 & $-1,2$ & \\
\hline & \multirow{3}{*}{7} & Recuento & 53 & 0 & 53 \\
\hline & & Frecuencia esperada & 52,1 & ,9 & 53,0 \\
\hline & & Residuos corregidos & 1,0 & $-1,0$ & \\
\hline
\end{tabular}




\begin{tabular}{|l|l|l|l|l|}
\hline \multirow{2}{*}{ Total } & Recuento & 1095 & 19 & 1114 \\
\cline { 2 - 5 } & Frecuencia esperada & 1095,0 & 19,0 & 1114,0 \\
\hline
\end{tabular}

Para la relación entre la variable enfermedad de los árboles en pie por rodal, al existir el 42,9\% de las casillas con valores esperados inferiores a 5, se aplicó el Test de Fisher, mismo que reportó un p-valor $=0,048<\alpha=0.05$, por tanto, se rechaza la hipótesis nula de independencia (Tabla 8).

Tabla 8. Pruebas de Chi-cuadrado de la tabla de contingencia de la variable enfermedad de los árboles en pie por rodal

\begin{tabular}{|l|l|l|l|l|l|l|}
\hline & Valor & gl & $\begin{array}{l}\text { Sig. } \\
\text { asintótica } \\
\text { (bilateral) }\end{array}$ & $\begin{array}{l}\text { Sig. exacta } \\
\text { (bilateral) }\end{array}$ & $\begin{array}{l}\text { Sig. exacta } \\
\text { (unilateral) }\end{array}$ & $\begin{array}{l}\text { Probabilidad } \\
\text { en el punto }\end{array}$ \\
\hline Chi-cuadrado de Pearson & $14,368^{\mathrm{a}}$ & 6 & 0,026 & 0,034 & & \\
\hline Razón de verosimilitudes & 18,828 & 6 & 0,004 & 0,004 & & \\
\hline Estadístico exacto de Fisher & 10,944 & & & 0,048 & & \\
\hline Asociación lineal por lineal & $10,296^{\mathrm{b}}$ & 1 & 0,001 & 0,001 & 0,000 & 0,000 \\
\hline N de casos válidos & 1114 & & & & & \\
\hline
\end{tabular}

a. 6 casillas $(42,9 \%)$ tienen una frecuencia esperada inferior a 5. La frecuencia mínima esperada es ,55.

b. El estadístico tipificado es $-3,209$.

\section{Análisis de contingencia de la relación de la variable debilidad de los árboles en pie por rodal}

El análisis de los residuos corregidos para la variable debilidad de los árboles en pie evidencia dependencia provocada por una tendencia al incremento en el rodal 2, 5, 6 y 7 en sus valores de ausencia $(3,0 ; 5,6 ; 3,3$ y $2,7)$ y decremento en sus valores de presencia $(-3,0 ;-5,6 ;-3,3$ y -2,7). En el caso de los rodales 1,3 y 4 ocurre lo contrario, hay un decremento en sus valores de ausencia $(-5,3 ;-3,4$ y $-3,4)$ e incremento en sus valores de presencia $(5,3 ; 3,4$ y 3,4 ; Tabla 9$)$.

Tabla 9. Análisis de contingencia de la relación de la variable debilidad de los árboles en pie por rodal

\begin{tabular}{|c|c|c|c|c|c|}
\hline & \multicolumn{2}{|c|}{ Debilidad } & \multirow{2}{*}{ Total } \\
\hline & & & 0 & 1 & \\
\hline \multirow{10}{*}{ Rodal } & \multirow{3}{*}{1} & Recuento & 327 & 75 & 402 \\
\hline & & Frecuencia esperada & 354,4 & 47,6 & 402,0 \\
\hline & & Residuos corregidos & $-5,3$ & 5,3 & \\
\hline & \multirow{3}{*}{2} & Recuento & 123 & 5 & 128 \\
\hline & & Frecuencia esperada & 112,8 & 15,2 & 128,0 \\
\hline & & Residuos corregidos & 3,0 & $-3,0$ & \\
\hline & \multirow{3}{*}{3} & Recuento & 22 & 10 & 32 \\
\hline & & Frecuencia esperada & 28,2 & 3,8 & 32,0 \\
\hline & & Residuos corregidos & $-3,4$ & 3,4 & \\
\hline & 4 & Recuento & 188 & 42 & 230 \\
\hline
\end{tabular}




\begin{tabular}{|l|l|l|l|l|l|}
\hline \multirow{1}{*}{} & Frecuencia esperada & 202,7 & 27,3 & 230,0 \\
\cline { 2 - 5 } & Residuos corregidos & $\mathbf{- 3 , 4}$ & $\mathbf{3 , 4}$ & \\
\cline { 2 - 5 } & Recuento & 192 & 0 & 192 \\
\cline { 2 - 5 } & Frecuencia esperada & 169,2 & 22,8 & 192,0 \\
\cline { 2 - 5 } & Residuos corregidos & $\mathbf{5 , 6}$ & $\mathbf{- 5 , 6}$ & \\
\cline { 2 - 5 } & Recuento & 77 & 0 & 77 \\
\cline { 2 - 5 } & Frecuencia esperada & 67,9 & 9,1 & 77,0 \\
\cline { 2 - 5 } & Residuos corregidos & $\mathbf{3 , 3}$ & $\mathbf{- 3 , 3}$ & \\
\cline { 2 - 5 } & Recuento & 53 & 0 & 53 \\
\cline { 2 - 5 } & Frecuencia esperada & 46,7 & 6,3 & 53,0 \\
\cline { 2 - 5 } & Residuos corregidos & $\mathbf{2 , 7}$ & $\mathbf{- 2 , 7}$ & \\
\hline \multirow{2}{*}{ Total } & Recuento & 982 & 132 & 1114 \\
\cline { 2 - 5 } & Frecuencia esperada & 982,0 & 132,0 & 1114,0 \\
\hline
\end{tabular}

Al aplicar el Test Chi-cuadrado de Pearson a los datos obtenidos de la Tabla 9 de contingencia, se rechaza la hipótesis nula y se acepta que hay dependencia entre las variables árboles débiles en pie y los rodales, al presentar un $\mathrm{p}$-valor $<\alpha=0.05$ (Tabla 10).

Tabla 10. Pruebas de Chi-cuadrado de la tabla de contingencia de la variable debilidad de los árboles en pie por rodal

\begin{tabular}{|l|l|l|l|l|l|l|}
\hline & Valor & gl & $\begin{array}{l}\text { Sig. } \\
\text { asintótica } \\
\text { (bilateral) }\end{array}$ & $\begin{array}{l}\text { Sig. exacta } \\
\text { (bilateral) }\end{array}$ & $\begin{array}{l}\text { Sig. exacta } \\
\text { (unilateral) }\end{array}$ & $\begin{array}{l}\text { Probabilidad } \\
\text { en el punto }\end{array}$ \\
\hline Chi-cuadrado de Pearson & $89,434^{\mathrm{a}}$ & 6 & 0,000 &.$^{\mathrm{b}}$ & & \\
\hline Razón de verosimilitudes & 123,272 & 6 & 0,000 &.$^{\mathrm{b}}$ & & \\
\hline Estadístico exacto de Fisher &.$^{\mathrm{b}}$ & & &.$^{\mathrm{b}}$ & & \\
\hline Asociación lineal por lineal & $36,671^{\mathrm{c}}$ & 1 & 0,000 & 0,000 & 0,000 & 0,000 \\
\hline N de casos válidos & 1114 & & & & & \\
\hline
\end{tabular}

a. 1 casillas $(7,1 \%)$ tienen una frecuencia esperada inferior a 5. La frecuencia mínima esperada es 3,79 .

b. No se puede efectuar el cálculo porque no hay suficiente memoria.

c. El estadístico tipificado es -6,056.

\section{Análisis de contingencia de la relación árboles moribundos en pie por rodal}

El análisis de los residuos corregidos para la variable árboles moribundos en pie por rodal, se evidencia dependencia provocada por una tendencia al incremento en el rodal 5 en su valor de ausencia $(2,7)$ y decremento en su valor de presencia $(-2,7)$. En el caso de los rodales 3 y 4 ocurre lo contrario, hay un decremento en sus valores de ausencia $(-3,4$ y $-3,8)$ e incremento en sus valores de presencia (3,4 y 3,8; Tabla 11). 
Tabla 11. Análisis de contingencia de la relación de árboles moribundos en pie por rodal

\begin{tabular}{|c|c|c|c|c|c|}
\hline & \multicolumn{2}{|c|}{ Moribundo } & \multirow{2}{*}{ Total } \\
\hline & & & 0 & 1 & \\
\hline \multirow{21}{*}{ Rodal } & \multirow{3}{*}{1} & Recuento & 386 & 16 & 402 \\
\hline & & Frecuencia esperada & 386,1 & 15,9 & 402,0 \\
\hline & & Residuos corregidos & ,0 & ,0 & \\
\hline & \multirow{3}{*}{2} & Recuento & 125 & 3 & 128 \\
\hline & & Frecuencia esperada & 122,9 & 5,1 & 128,0 \\
\hline & & Residuos corregidos & 1,0 & $-1,0$ & \\
\hline & \multirow{3}{*}{3} & Recuento & 27 & 5 & 32 \\
\hline & & Frecuencia esperada & 30,7 & 1,3 & 32,0 \\
\hline & & Residuos corregidos & $-3,4$ & 3,4 & \\
\hline & \multirow{3}{*}{4} & Recuento & 211 & 19 & 230 \\
\hline & & Frecuencia esperada & 220,9 & 9,1 & 230,0 \\
\hline & & Residuos corregidos & $-3,8$ & 3,8 & \\
\hline & \multirow{3}{*}{5} & Recuento & 191 & 1 & 192 \\
\hline & & Frecuencia esperada & 184,4 & 7,6 & 192,0 \\
\hline & & Residuos corregidos & 2,7 & $-2,7$ & \\
\hline & \multirow{3}{*}{6} & Recuento & 77 & 0 & 77 \\
\hline & & Frecuencia esperada & 74,0 & 3,0 & 77,0 \\
\hline & & Residuos corregidos & 1,8 & $-1,8$ & \\
\hline & \multirow{3}{*}{7} & Recuento & 53 & 0 & 53 \\
\hline & & Frecuencia esperada & 50,9 & 2,1 & 53,0 \\
\hline & & Residuos corregidos & 1,5 & $-1,5$ & \\
\hline \multirow{2}{*}{ Total } & & Recuento & 1070 & 44 & 1114 \\
\hline & & Frecuencia esperada & 1070,0 & 44,0 & 1114,0 \\
\hline
\end{tabular}

La relación entre la variable árboles moribundos en pie por rodal, dada la existencia del $21,4 \%$ de las casillas con valores esperados inferiores a 5, se aplicó el Test de Fisher, mismo que reportó un p-valor $<\alpha=0.05$, por tanto, se rechaza la hipótesis nula de independencia (Tabla 12).

Tabla 12. Pruebas de Chi-cuadrado de la tabla de contingencia de la variable árboles moribundos en pie por rodal

\begin{tabular}{|l|l|l|l|l|l|l|}
\hline & Valor & gl & $\begin{array}{l}\text { Sig. } \\
\text { asintótica } \\
\text { (bilateral) }\end{array}$ & $\begin{array}{l}\text { Sig. exacta } \\
\text { (bilateral) }\end{array}$ & $\begin{array}{l}\text { Sig. exacta } \\
\text { (unilateral) }\end{array}$ & $\begin{array}{l}\text { Probabilidad } \\
\text { en el punto }\end{array}$ \\
\hline Chi-cuadrado de Pearson & $34,933^{\mathrm{a}}$ & 6 & 0,000 & 0,000 & & \\
\hline Razón de verosimilitudes & 36,254 & 6 & 0,000 & 0,000 & & \\
\hline Estadístico exacto de Fisher & 30,524 & & & 0,000 & & \\
\hline Asociación lineal por lineal & $2,216^{\mathrm{b}}$ & 1 & 0,137 & 0,146 & 0,073 & 0,011 \\
\hline N de casos válidos & 1114 & & & & & \\
\hline
\end{tabular}

a. 3 casillas $(21,4 \%)$ tienen una frecuencia esperada inferior a 5. La frecuencia mínima esperada es 1,26.

b. El estadístico tipificado es $-1,489$. 


\section{Análisis de contingencia de la relación árboles muertos en pie por rodal}

El análisis de los residuos corregidos para la variable árboles muertos en pie por rodal no procede por cuanto, no existe dependencia alguna a un nivel de confianza de $95 \%$ tal y como lo ratifica el Test de Fisher con un pvalor $=0,232>\alpha=0.05$, que se muestra en la Tabla 13.

Tabla 13. Análisis de contingencia de la relación árboles muertos en pie por rodal

\begin{tabular}{|c|c|c|c|c|c|}
\hline & \multicolumn{2}{|c|}{ Muertos en pie } & \multirow{2}{*}{ Total } \\
\hline & & & 0 & 1 & \\
\hline \multirow{21}{*}{ Rodal } & \multirow{3}{*}{1} & Recuento & 395 & 7 & 402 \\
\hline & & Frecuencia esperada & 396,2 & 5,8 & 402,0 \\
\hline & & Residuos corregidos &,- 6 & 6 & \\
\hline & \multirow{3}{*}{2} & Recuento & 125 & 3 & 128 \\
\hline & & Frecuencia esperada & 126,2 & 1,8 & 128,0 \\
\hline & & Residuos corregidos &,- 9 & ,9 & \\
\hline & \multirow{3}{*}{3} & Recuento & 32 & 0 & 32 \\
\hline & & Frecuencia esperada & 31,5 & ,5 & 32,0 \\
\hline & & Residuos corregidos & ,7 &,- 7 & \\
\hline & \multirow{3}{*}{4} & Recuento & 224 & 6 & 230 \\
\hline & & Frecuencia esperada & 226,7 & 3,3 & 230,0 \\
\hline & & Residuos corregidos & $-1,7$ & 1,7 & \\
\hline & \multirow{3}{*}{5} & Recuento & 192 & 0 & 192 \\
\hline & & Frecuencia esperada & 189,2 & 2,8 & 192,0 \\
\hline & & Residuos corregidos & 1,8 & $-1,8$ & \\
\hline & \multirow{3}{*}{6} & Recuento & 77 & 0 & 77 \\
\hline & & Frecuencia esperada & 75,9 & 1,1 & 77,0 \\
\hline & & Residuos corregidos & 1,1 & $-1,1$ & \\
\hline & \multirow{3}{*}{7} & Recuento & 53 & 0 & 53 \\
\hline & & Frecuencia esperada & 52,2 & 8 & 53,0 \\
\hline & & Residuos corregidos & ,9 &,- 9 & \\
\hline \multirow{2}{*}{\multicolumn{2}{|c|}{ Total }} & Recuento & 1098 & 16 & 1114 \\
\hline & & Frecuencia esperada & 1098,0 & 16,0 & 1114,0 \\
\hline
\end{tabular}

La relación entre la variable árbol muerto en pie por rodal, al existir el 42,9\% de las casillas con valores esperados inferiores a 5, se aplicó el Test de Fisher, mismo que reportó un $\mathrm{p}$-valor $=0,232>\alpha=0.05$, por tanto, se acepta la hipótesis de nulidad que plantea independencia entre las variables a un nivel de significancia 5\% (Tabla 14). 
Tabla 14. Pruebas de Chi-cuadrado de la tabla de contingencia de la variable árbol muerto en pie por rodal

\begin{tabular}{|l|l|l|l|l|l|l|}
\hline & Valor & gl & $\begin{array}{l}\text { Sig. asintótica } \\
\text { (bilateral) }\end{array}$ & $\begin{array}{l}\text { Sig. exacta } \\
\text { (bilateral) }\end{array}$ & $\begin{array}{l}\text { Sig. exacta } \\
\text { (unilateral) }\end{array}$ & $\begin{array}{l}\text { Probabilidad } \\
\text { en el punto }\end{array}$ \\
\hline Chi-cuadrado de Pearson & $8,401^{\mathrm{a}}$ & 6 & 0,210 & 0,199 & & \\
\hline Razón de verosimilitudes & 12,916 & 6 & 0,044 & 0,048 & & \\
\hline Estadístico exacto de Fisher & 6,909 & & & 0,232 & & \\
\hline Asociación lineal por lineal & $2,712^{\mathrm{b}}$ & 1 & 0,100 & 0,108 & 0,055 & 0,014 \\
\hline N de casos válidos & 1114 & & & & & \\
\hline
\end{tabular}

a. 6 casillas $(42,9 \%)$ tienen una frecuencia esperada inferior a 5. La frecuencia mínima esperada es ,46. b. El estadístico tipificado es -1,647.

Los indicadores de salud obtenidos, al evaluar la plantación de eucalipto mostraron el comportamiento de los rodales escogidos para su análisis (Sepúlveda, 2002), estos evidencian la influencia de ciertos factores del suelo como la estructura masiva que poseen los suelos del bosque plantado en estudio (Guallpa et al., 2016), parámetro que limita el desarrollo del sistema radicular y por ende incide en la forma del fuste y distribución de su biomasa (ramas y follaje) pueden reflejar el estado general de salud del rodal (Cumming, Nowak, Twardus, Hoehn, Mielke, \& Rideout, 2007; Zarnoch, Bechtold, \& Solte, 2004). De acuerdo a lo señalado, los principales elementos de la productividad primaria son las ramas y follaje que conforman la copa de un árbol, púes copas simétricas y densamente foliadas están asociadas a un alto potencial fotosintético (Randolph, 2004; Randolph, Rose, Oswalt, \& Brown, 2013; Awal et al., 2010), mientras que las copas pequeñas y dispersas apuntan a condiciones desfavorables de crecimiento debido a condiciones de sitio no óptimas por competencia, estrés hídrico, compactación de suelo, bajos niveles de boro, entre otros, son factores que promueven la susceptibilidad de los árboles al ataque de enfermedades, y se manifiestan severos desórdenes en el crecimiento (Lehto, Ruuholaa, \& Dellb, 2010; Zarnoch et al., 2004) y son típicas de árboles en declinación cuya apariencia y condición respecto a la cantidad y distribución del follaje proporcionan una idea clara de la historia de vida de cada individuo y del ambiente donde se está desarrollando (Nadolny, 1995).

\section{Agrupamiento del estado de salud de los árboles en pie por rodal}

Para el análisis del estado de salud de los rodales (Tabla 15), se procedió a crear grupos de rodales que pudieran manejarse de forma homogénea en cuanto a un tratamiento silvicultural. 
Tabla 15. Frecuencias de presencia de los seis criterios evaluados para la variable salud

\begin{tabular}{|c|c|c|c|c|c|c|}
\hline Rodales & Vigorosidad & Saludable & Enfermedad & Debilidad & Moribundo & Muerto en pie \\
\hline 1 & 0 & 0,721393 & 0,034826 & 0,186567 & 0,039801 & 0,017413 \\
\hline 2 & 0 & 0,90625 & 0,007813 & 0,039063 & 0,023438 & 0,023438 \\
\hline 3 & 0 & 0,53125 & 0 & 0,3125 & 0,15625 & 0 \\
\hline 4 & 0,086957 & 0,6 & 0,017391 & 0,182609 & 0,082609 & 0,026087 \\
\hline 5 & 0 & 1 & 0 & 0 & 0,005208 & 0 \\
\hline 6 & 0 & 1 & 0 & 0 & 0 & 0 \\
\hline 7 & 0 & 1 & 0 & 0 & 0 & 0 \\
\hline
\end{tabular}

Se conformó los distintos conglomerados, usando para ello conglomerados jerárquicos, vinculación de Ward y distancias Euclideas al cuadrado (Di Rienzo et al., 2009). Los resultados se muestran en el Dendrograma (Figura 2) de aquellos rodales con similares atributos de salud corresponden a los rodales 2, 5, 6 y 7 en un primer grupo, cuya agrupación a excepción del rodal 2 , en su mayoría coincide con el estudio de productividad que tipifica a los rodales 5,6 y 7 de aprovechamiento en la plantación de $E$. globulus, según lo reportado por Guallpa et al.(2016). Así mismo se conformó un segundo grupo formado por los rodales 1,3 y 4, cuya categorización de rodales se inserta dentro del área para conservación que asocia a los rodales 1 , 2, 3 y 4 según lo definido al considerar el rendimiento de cada rodal (Guallpa et al., 2016). Con base a la información disponible en salud forestal de los rodales evaluados (Rondeux, 2010), es viable planificar una estrategia de manejo silvícola sostenible de la plantación en estudio para el área de aprovechamiento conformada por los rodales 2, 5, 6 y 7 (FONAM, 2007). Mediante la ejecución de cortas de aprovechamiento de los árboles y posterior manejo de los rebrotes a partir de un plan operativo y táctico (Musálem, 2006), y la ejecución de actividades de carácter conservador a fin de garantizar su uso actual y permanente para los rodales 1, 3 y 4 (Guallpa et al., 2019).

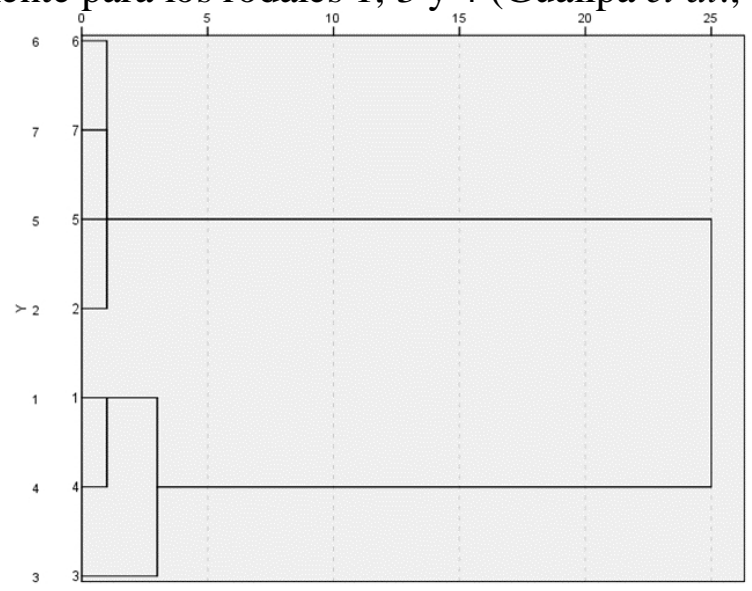

Figura 2. Dendrograma resultante del análisis de conglomerados para el estado de de salud de los árboles (método de Ward, distancia Euclidea al cuadrado). 


\section{Conclusione}

Los resultados del estado de salud forestal de los rodales que integran el bosque plantado de E. globulus, reportó cinco asociaciones significativas para la mayoría de los criterios considerados en la evaluación a excepción del rasgo árbol muerto en pie por rodal, lo cual indica la interacción entre las características del sitio y la especie forestal en estudio.

La creación de grupos de rodales simplificó la planificación del manejo de la plantación, reduciendo a dos estrategias silvícolas que comprenderían a todos los rodales incorporados en cada cluster desde el punto de vista del estado de salud forestal, mismo que influye en la productividad, pero a su vez sustenta el manejo técnico de la plantación de eucalipto considerando la ejecución del aprovechamiento de los rodales 2, 5, 6 y 7. De acuerdo a los resultados determinados para el estado de salud forestal de los rodales evaluados, se obtendrá madera de calidad 1 (árboles vigorosos), de calidad 2 (árboles saludables), de calidad 3 (árboles enfermos) y madera de calidad 4 (árboles débiles y moribundos) y con enfoque conservacionista a los rodales 1,3 y 4 .

\section{Agradecimiento}

Los autores agradecen a la ESPOCH, y la Facultad de Recursos Naturales por la oportunidad que otorga a los profesionales y estudiantes del área forestal, al brindar facilidades que permite llevar a cabo investigaciones, cuyos resultados contribuyen a la toma de decisiones técnicas orientadas a un manejo sostenible de los recursos forestales, a la Ing. Sonia Rosero H. por sus valiosas críticas que nos hizo durante la preparación del manuscrito.

\section{References:}

1. Awal, M. A., Ohta, T., Matsumoto, K., Toba, T., Daikoku, K., Hattori, S., Hiyama, T., \& Park, H. (2010). Comparing the carbon sequestration capacity of temperate deciduous forests between urban and rural landscapes in central Japan. Urban Forestry and Greening, 9, 261-270.

2. Cumming, B. A., Nowak, D. J., Twardus, D. B., Hoehn, R., Mielke, M., \& Rideout, R. (2007). Urban forest of Wisconsin: Pilot Monitoring Project 2002. (NA-FR-05-07) USDA Forest Service, Northeastern Area. National Forest Health Monitoring Program.

3. Dale, V. H., Joyce, L. A., McNulty, S., Neilson, R. P., Ayres, M. P., Flannigan, M. D., Hanson, P. J., Irland, L. C., Lugo, A. E., Peterson, C. J., Simberloff, D., Swanson, F. J., Stocks, B. J., \& Wotton, B. M. (2001). Climate change and forest disturbances. Bioscience, 51(9): 723-734.

4. De la Fuente-Fernández, S. (2011). Tablas de Contingencia. Facultad de Ciencias_Económicas y Empresariales. Universidad Autónoma de 
Madrid.

Recuperado

de:

http://www.estadistica.net/ECONOMETRIA/CUALITATIVAS/CO

NTINGENCIA/tablas-contingencia.pdf

5. De la Fuente-Fernández, S. (2011a). Análisis de Conglomerados. Facultad de Ciencias_Económicas y Empresariales. Universidad Autónoma de Madrid. Recuperado de: http://www.fuenterrebollo.com/Economicas/ECONOMETRIA/SEG MENTACION/CONGLOMERADOS/conglomerados.pdf

6. Di Rienzo, J.A., Casanoves, F., Balzarini, M.G., Conzalez, L., Tablada, M., \& Robledo, C.W. (2009). InfoStat versión 2009. Grupo InfoStat, FCA, Universidad Nacional de Córdoba.

7. ETIFN. (2014). Manual de Campo. Procedimientos para la planificación, medición y registro de información del Inventario Forestal Nacional de Paraguay. FAO-PNUD-PNUMAINFONASEAM-FAPI.

8. FAO. (2011). Diseños de muestreo de las Evaluaciones Forestales Nacionales.

Recuperado

de:

http://www.fao.org/fileadmin/user_upload/national_forest_assessmen t/images/PDFs/Spanish/KR2_ES_4_.pdf

9. Fondo Nacional del Ambiente. FONAM. (2007). Guía práctica para la instalación y manejo de plantaciones forestales. Lima, Perú.

10. García, G. (2016). Investigación comercial (4 ${ }^{\text {ta }}$ ed.). España: Esic.edu/editorial.

11. Granados, D., \& López, G. (2001). Declinación forestal. Revista Chapingo Serie Ciencias Forestales y del Ambiente, 7(1), 5-13

12. Guallpa, M., Rosero, S., Samaniego, M., \& Cevallos, E. (2016). Caracterización edáfica y dasométrica de una plantación de Eucalyptus globulus Labill y propuesta de manejo en la zona estepa espinosa Montano Bajo, Riobamba, Ecuador. Enfoque UTE, 7(3), 2640.

13. Guallpa, M., Rosero, S., Montenegro, G., \& Quinchuela, D (2018). Estimación de los residuos forestales en los aserraderos de tres cantones, Zona 3 interandina, Ecuador. European Scientific Journal, 14(30), 228-239.

14. Guallpa, M., Lara, N., Espinoza, M., Guilcapi, E., \& Fosado, O. (2019). Valoración cualitativa de una plantación de Eucalyptus globulus Labill en el sector de Licto, Riobamba, Ecuador. Polo del Conocimiento, 4(4), 126-152.

15. Grupo Empresarial ENCE. (2009). La Gestión Forestal Sostenible y el Eucalipto. España: ENCE.

16. Haberman, S. J. (1978). Analysis of Quantitative Data. Volume 1 Introductory Topics 
17. Hernández, J. (2014). Curso Regional de Regulación, Manejo y Salud Forestal. Chihuahua

18. Jiménez, C. (2008). Calidad y valoración de plantaciones forestales: aplicación práctica en cinco plantaciones de Vochysia guatemalensis Donn. Sm. (cebo) en las zonas Norte y Atlántica de Costa Rica. Kurú: Revista Forestal, 5(15): págs. 5

19. Lehto, T., Ruuholaa, T., \& Dellb, B. (2010). Boron in forest trees and forest ecosystems. Forest Ecology and Management, 260, 2053-2069. doi: $10.1016 /$ j.foreco.2010.09.028

20. López, P., \& Fachelli, S. (2015). Metodología de la Investigación Social Cuantitativa. Departament de Sociologia. Universitat Autónoma de Barcelona. Recuperado de https://ddd.uab.cat/pub/caplli/2015/131469/metinvsoccuan_cap36a2015.pdf

21. Ministerio de Agricultura, Ganadería, Acuacultura y Pesca. MAGAP. (2015). Programa de Incentivos para la Reforestación con Fines Comerciales. Guayaquil, Ecuador: MAGAP.

22. Murillo, O., \& Badilla, Y. (2010). Calidad de la plantación forestal. ITCR, Cartago, C.R. 67 p.

23. Musálem, M. (2006). Silvicultura de Plantaciones Forestales Comerciales. Chapingo, México: Universidad Autónoma Chapingo División de Ciencias Forestales.

24. Nadolny, C. (1995). Causes of tree decline /dieback in NSW. En A. Kater (Ed.), Redressing rural tree decline in NSW: proceedings of the 'After Dieback' conference presented by Greening Australia, Orange Civic Centre (pp. 11-18). Orange, NSW: Greening Australia.

25. Nájera, J. A., \& Hernández, H. E. (2008). Relaciones morfométricas de un bosque coetáneo de la región del Salto, Durango. Ra Ximhai, 4 (1), 69-81.

26. Nel, L. (2014). Estadística con SPSS 22. Lima: Macro.

27. Randolph, K. C. (2004). An evaluation of change in tree crown characteristics to assess forest health in two Indiana State Parks. Northern Journal of Applied Forestry, 21(1), 50-55

28. Randolph, K. C., Rose, A. K., Oswalt, C. M., \& Brown, M. J. (2013). Status of black walnut (Juglans nigra L.) in the eastern United States of the discovery of thousand cankers disease. Southern Appalachian Botanical Society, 78(1), 2-14.

29. Rojas, O., \& Murillo, O. (2000). Calidad de las plantaciones de teca en la península de Nicoya, Costa Rica. Agronomía costarricense, 24(2), 65-75 p.

30. Rondeux, J. (2010). Medición de árboles y masas forestales. España: Mundi-Prensa. 
31. Saavedra, L., Alvarado, D., Hernández, P., Martínez, T., Mora, G., \& Villa, J. (2016). Condición de copa, indicador de salud en árboles urbanos del Bosque San Juan de Aragón, Ciudad de México. Madera y Bosques, 22(2), 15-27.

32. Salazar, M. (2008). Propuesta de procedimientos para el establecimiento y seguimiento de parcelas permanentes de medición forestal en plantaciones beneficiarias del PINFOR. Unidad de Fomento Y Desarrollo Forestal, INAB. Guatemala.

33. Scharenbroch, B. C., Lloyd, J. E., \& Johnson, J. L. (2005). Distinguising urban environments with physical, chemical and biological soil properties. Pedobiologia, 49, 283-296.

34. Schomaker, M. E., Zarnoch, S. J., Bechtold, W. A., Latelle, D. J., Burkman, W. G., \& Cox, S. M. (2007). Crown-condition classification: A guide to data collection and analysis. (General Technical Report SRS-102) USDA Forest Service.

35. Sepúlveda, S. (2002). Desarrollo sostenible micro regional. Métodos para planificación local. San José, CR, IICA-UNA-CDT. 312 p.

36. Sierra , R., Cerón, C., Palacios, W., \& Valencia, R. (1999). Propuesta preliminar de un sistema de clasificación para el Ecuador Continental. Quito, Ecuador: Proyecto INEFAN/GEF-BIRF y EcoCiencia.

37. Zarnoch, S. J., Bechtold, W. A., \& Solte, K. W. (2004). Using crown condition variables as indicators of forest health. Canadian Journal of Forest Research, 34, 1057-1070. 